\title{
DENTSPLY DETREY
}

\section{Endlich eine Füllungstherapie ohne Zeit raubende Technik}

Ein Highlight markierte im Rahmen des 24.

Berliner Zahnärztetages die Einführung des neuen Komposits SDR Smart Dentin Replacement von DENTSPLY DETREY.

Die Handhabung des neuen Komposits ist denkbar einfach: Zunächst wird die Kavität, wie gewohnt, mit einem Bonding vorbereitet, wobei SDR mit allen konventionellen Methacrylatbasierten Adhäsiven kompatibel ist. Es lässt sich anschließend in fließfähiger Form bis zu 4 mm zügig einfüllen und fließt an die Kavitätenwände an - komplexe und zeitaufwändige Schichttechnik wird damit überflüssig. Unregelmäßigkeiten auf dem Kavitätenboden sowie unter sich gehende Bereiche werden einfach und problemlos aufge- füllt. Die Oberfläche glättet sich von selbst, so dass man für diesen Arbeitsschritt kein Modellierinstrument mehr benötigt. Nach dem Aushärten wird die letzte Schicht mit einem beliebigen Komposit in der passenden Zahnfarbe modelliert.

Durch ein neuartiges, patentiertes Monomer wird der Schrumpfungsstress bei der Aushärtung stark reduziert. Damit empfehlen sowohl das einfache Handling als auch die physikalischen Eigenschaften das neue Komposit als Material für die Grundversorgung im zahnärztlichen Praxisalltag.

ス Tel. 08000 - 735000 (kostenfrei)

info@dentsply.de

www.dentsply.de

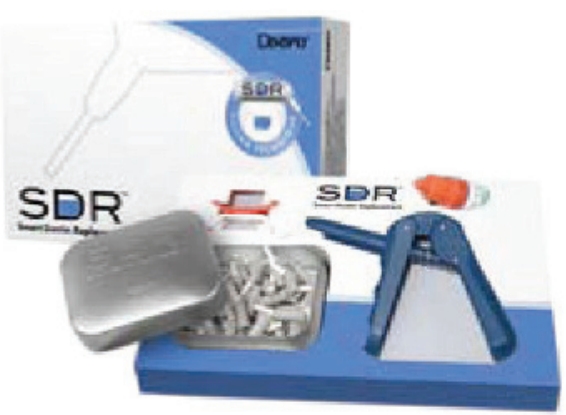

\section{Pharmatechnik}

\section{2-D Röntgen statt Körperscanner}

Klarer Durchblick ganz ohne Körperscanner - dafür sorgt LinuDent mit dem „Veraview IC-5"-Röntgengerät. Und das Beste, dieses Rönt-

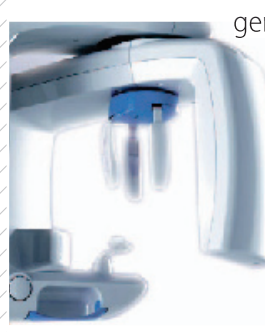

gengerät muss nicht einmal gekauft werden um bei den Praxen zum Einsatz zu kommen.

"Detailreiche Röntgenbilder in Hochauflösung sind heute in der Zahnarztpraxis unerläss- lich. Mit unserem IC-5 bieten wir den Zahnärzten ein Gerät zu einem hervorragenden Preis-Leistungsverhältnis, das Röntgenbilder in herausstechender Qualität liefert", erklärt Holger Reinhoff, Geschäftsleiter Vertrieb bei LinuDent. In nur 5,5 Sekunden fertigt das 2-D-Röntgengerät die Bilder. Dadurch sind die Patienten einer sehr kurzen und geringen Strahlenbelastung ausgesetzt.

LinuDent bietet den Zahnärzten eine interessante Finanzierungsmöglichkeit für die HighTech-Geräte. „Modernste Praxisausstattung ohne
Kreditaufnahme ist mit unserem Mietkonzept kein Problem mehr. Die Geräte können bei uns gemietet werden und diesen monatlichen Betrag als feste Kosten von der Steuer absetzen. Die Aufwendungen für Technikereinsätze und Updates sind mit der Miete bereits abgedeckt", erläutert Reinhoff.

入 Tel. 08151 - 4442-0 info@linudent.de www.linudent.de

\section{Humanchemie}

\section{Interims-Kronenzement - jetzt neu mit Dosierhilfe}

Da immer mehr Patienten allergische Reaktionen auf Kunststofffüllungen zeigen und trotzdem amalgamfreie Versorgungen wünschen, steigt der Bedarfan Zementen als alternatives Füllungs- und Befestigungsmaterial. Häufig erweist es sich jedoch als schwierig eine gleichbleibende Festigkeit bei jedem Anrührvorgang zu erzielen. Daher wird der Interims-Kronenzement Cupral $^{\circledR}$ von Humanchemie ab sofort mit einer Dosierhilfe - bestehend aus einem Dosierlöffel und einem Tropfer - ausgeliefert.
Der Interims-Kronenzement ist ein Eugenol-freier alkalischer Unterfüllungs- und Heilzement. Nach dem Anrühren mit Aqua dest. bindet er langsam durch Hydrolyse unter Bildung von Calciumhydroxid ab. Er bleibt dabei von geringer Festigkeit, die durch das Mischungsverhältnis zu Zement variierbar ist.

Auf die Pulpa wirkt er beruhigend, da das alkalische Calciummilieu die bei der Präparation entstandenen initialen Entzündungsreize paralysiert und dabei die Sekundärdentinbildung stimuliert.

Zusätzlich zur alkalischen Proteolyse wirkt auch die schwache Kupfer-Dotierung desinfizierend.
Das Produkt findet so Anwendung beim temporären Aufsetzen von Kronen und leichteren Brücken, sowie als beruhigende Unterfüllung für die Pulpa.

入 Tel. 05181 - 24633

info@humanchemie.de www.humanchemie.de 